In memory of Henri Rukhadze

\title{
NON-COULOMB NUCLEAR REACTIONS OF TRANSATOMS. STELLAR ENERGY AND NUCLEOSYNTHESIS
}

\author{
Gennady V. Mishinsky \\ Joint Institute for Nuclear Research, http://www.jinr.ru \\ Dubna 141980, Moscow Region, Russian Federation \\ mysh@jinr.ru
}

Abstract. The exchange Coulomb energy of two charged particles paired in orthoboson $(\mathrm{S}=\uparrow \uparrow, 1$, $2, \ldots)$ has the character of attraction and it completely compensates, in the first order of perturbation theory, the energy of their Coulomb repulsion. For strongly interacting identical particles, including atomic nuclei, this fact leads to the launch of nuclear reactions without Coulomb barrier. This explains the feasibility of low energy nuclear reactions. Consequently, there is a stellar energy source as an alternative to thermonuclear fusion, and there is a process of nucleosynthesis as an alternative to the explosion of supernovae.

Keywords: quantum physics, exchange interaction, spin Bose-Einstein condensate, nuclear fusion, low energy nuclear reactions, stellar energy source, nucleosynthesis, atomic-nuclear science in condensed matter

PACS: 36.10.-k; 32.10.-f; 76.30.-v; 03.75.Mn; 03.75.Nt; 42.55.Vc; 25.60.Pj; 26.20.Cd; 26.20.Fj

Bibliography -25 references

Received 13.04.2018

RENSIT, 2018, 10(1):35-52

DOI: $10.17725 /$ rensit.2018.10.035

\section{CONTENTS}

1. INTRODUCTION (35)

2. EXCHANGE INTERACTION OF ELECTRONS IN A HELIUM ATOM (36)

3. EXChANGE INTERACTION OF ELECTRONS IN A HELIUM TRANSATOM (37)

4. EXCHANGE INTERACTION OF ELECTRONS IN A HYDROGEN MOLECULE (39)

5. INTERACTION OF ELECTRONS IN HYDROGEN atoms in Strong Magnetic FIEld. The FORMATION OF A HYDROGEN TRANSMOLECULE (40)

6. Nuclear reactions without a Coulomb BARRIER (42)

7. EXPERIMENTS (45)

8. Stellar energy and NuCleosynthesis (49)

9. Conclusion (50)

References (50)

\section{INTRODUCTION}

The transatom is an atom that has an electron Bose-Einstein condensate [1-3]. In the transatom the electrons, not necessarily all, are in a paired state. Unlike superconductors $S=0$, a pair of electrons in the transatom constitute an orthoboson $S=1$. Such pairing of two electrons takes place in a strong magnetic field [1], due, first, to the exchange interaction of electrons having the character of attraction, and, secondly, to the appearance of correlated electron oscillations near their orbitals that generate for them a new quantum number $n_{b}$. In the orthoboson, electrons have oscillating quantum numbers, which are opposite in sign $n_{b}^{1}=-n_{b}^{2}, n_{b}=1,2,3, \ldots$ Therefore, the Pauli principle is satisfied for them. Trajectories of electron motion are closed spirals enclosed in each other located on the surface of toroid.

In this paper, we consider the formation of light transatoms, with a charge of the nucleus $Z<$ 6 in a strong magnetic field and their interaction. The interaction of identical transatoms leads not only to the creation of transmolecules, but also to the realization of low energy nuclear reactions.

In coupled systems: electrons in atoms and molecules can not be, according to the Pauli principle, in the same quantum state. This condition for two electrons in a helium atom and a hydrogen molecule is expressed in the fact that their total wave function must be an antisymmetric function, 
and it must remain so after the permutation of electrons. A symmetric wave function can not be used to describe the two electrons.

The total wave function of two electrons is usually represented as the product of spin wave function of electrons by the wave function of their spatial motion. The spin wave function of an electron can take on two values: that with a positive projection of the spin on the selected direction $S^{+}$and that with a negative projection of the spin on the selected direction $S^{-}$. The spin function of two electrons can be represented by the product of the spin functions of individual electrons or by a combination of their product. Due to the identity of electrons, their common spin function in the interchange of electrons must have a defined symmetry: to be symmetric or antisymmetric. Symmetric spin wave functions describe the triplet state of two electrons $(S=$ $\left.1, m_{S}=+1,0,-1\right)$, and antisymmetric spin wave functions describe the singlet state $(S=0)$.

The wave function of the spatial motion of two electrons must satisfy the same requirements as the spin function of two electrons. If the wave function of the spatial motion of the first electron in the state $a$ is $\Psi_{a}(1)$ and $\Psi_{b}(2)$ is the wave function of the spatial motion of the second electron in state $b$, then the symmetric wave function $\Psi^{+}(1,2)$ and the antisymmetric wave function $\Psi^{-}(1,2)$ can be represented as:

$$
\begin{gathered}
\Psi^{+}(1,2)=\Psi_{a}(1) \Psi_{b}(2)+\Psi_{a}(2) \Psi_{b}(1), \\
\Psi^{-}(1,2)=\Psi_{a}^{(}(1) \Psi_{b}(2)-\Psi_{a}^{(}(2) \Psi_{b}(1) .
\end{gathered}
$$

Since the total wave function of two electrons must be antisymmetric function, and the product of symmetric function by antisymmetric function is an antisymmetric function, only the following combinations are acceptable [4]:

$$
\begin{aligned}
& {\left[\Psi_{a}(1) \Psi_{b}(2)+\Psi_{a}(2) \Psi_{b}(1)\right] \times\left[S^{+}(1) S^{-}(2)-S^{+}(2) S^{-}(1)\right] ;(2)} \\
& {\left[\Psi_{a}(1) \Psi_{b}(2)-\Psi_{a}(2) \Psi_{b}(1)\right] \times\left[S^{+}(1) S^{+}(2)\right] ;} \\
& {\left[\Psi_{a}(1) \Psi_{b}(2)-\Psi_{a}(2) \Psi_{b}(1)\right] \times\left[S^{-}(1) S^{-}(2)\right] ;} \\
& {\left[\Psi_{a}(1) \Psi_{b}(2)-\Psi_{a}(2) \Psi_{b}(1)\right] \times\left[S^{+}(1) S^{-}(2)+S^{+}(2) S^{-}(1)\right] ;(3 c) .}
\end{aligned}
$$

The wave function of to electrons $\Psi_{a}$ and $\Psi_{b}$ are written without taking into account their interaction and correspond to the eigenvalues of the energy $E_{a}$ and $E_{b}$. They are well known from the theory of hydrogen-like atoms. In this case, the system energy is equal to $E=E_{a}+$ $E_{b}$. The electron interaction is accounted for by perturbation theory.

\section{EXCHANGE INTERACTION OF ELECTRONS IN HELIUM ATOM}

The perturbation energy between electrons in a helium atom is equal to [4]:

$V=k \cdot e^{2} / r_{1,2}$

where $r_{1,2}-$ distance between electrons, $\mathrm{k}=\left(4 \pi \varepsilon_{0}\right)^{-1}$ $=8.99 \cdot 10^{9} \mathrm{~N} \cdot \mathrm{m}^{2} / \mathrm{C}^{2}\left(\varepsilon_{0}=8.85 \cdot 10^{-12} \mathrm{~F} / \mathrm{m}-\right.$ electric constant), $e=1.6022 \cdot 10^{-19} \mathrm{C}$ - electron charge.

The first correction to system energy is calculated in the following way:

$\Delta E^{(1)}=\frac{\int \psi^{*}(1,2)\left[k \cdot e^{2} / r_{12}\right] \psi(1,2) d V_{1} d V_{2}}{\int \psi^{*}(1,2) \psi(1,2) d V_{1} d V_{2}}$,

where $d V_{1}=d x_{1} d y_{1} d \Sigma_{1}, d V_{2}=d x_{2} d y_{2} d \Sigma_{2}$, and $\psi(1,2)$ - corresponds to wave functions (2 and 3). The integral in the denominator is the normalization integral. Since the perturbation energy $V$ does not depend on the spin variables, the same factors appear in the numerator and denominator at their averaging, which are reduced. As a result, $\psi(1,2)$ corresponds to parts of the wave functions (2 and 3), which depend on the coordinates only. Consequently,

$$
\begin{aligned}
& \psi^{*}(1,2) \psi(1,2)=\psi_{a}^{*}(1) \psi_{b}^{*}(2) \psi_{a}(1) \psi_{b}(2)+ \\
& +\psi_{a}^{*}(2) \psi_{b}^{*}(1) \psi_{a}(2) \psi_{b}(1) \pm \\
& \pm\left[\psi_{a}^{*}(1) \psi_{b}^{*}(2) \psi_{a}(2) \psi_{b}(1)+\right. \\
& \left.+\psi_{a}(1) \psi_{b}(2) \psi_{a}^{*}(2) \psi_{b}^{*}(1)\right] .
\end{aligned}
$$

The integrals of first two terms of (6) are equal to each other. They are equal in the numerator (5), since the interaction of electrons is symmetrical with respect to their coordinates. We introduce the notations $C$ and $A$ :

$$
C=\int\left|\psi_{a}(1)\right|^{2}\left[k \cdot e^{2} / r_{12}\right]\left|\psi_{b}(2)\right|^{2} d V_{1} d V_{2} .
$$

The integral $C$ is responsible for the ordinary, average Coulomb energy of interaction between electron clouds.

The integral $A(8)$ of the terms in square brackets (6) is the exchange integral arising from electron identity. That energy is called exchange energy [5]. 
$A=\frac{1}{2} \int\left[\psi_{a}^{*}(1) \psi_{b}^{*}(2) \psi_{a}(2) \psi_{b}(1)+\psi_{a}(1) \psi_{b}(2) \psi_{a}^{*}(2) \psi_{b}^{*}(1)\right] \times$

$\times\left[k \cdot e^{2} / r_{12}\right] d V_{1} d V_{2}$.

Thus, the correction to energy can be represented as:

$\Delta E^{(1)}=C \pm A, a \neq b$,

$\Delta E^{(1)}=C+A, a=b$.

PARAHELIUM. The plus sign refers to the singlet state when electron spins are directed opposite to each other $(S=0)$, the states are: $E_{a}+E_{b}+C+A$ and $2 E_{a}+C+A$ (Fig. 1). The ground parahelium state $2 E_{a}+C+A, 1^{1} S_{0}$ has the energy $79.0 \mathrm{eV}$, the first excited parahelium state $-E_{a}+E_{b}+C+A$, $2^{1} S_{0}-58.4 \mathrm{eV}$.

ORTHOHELIUM. The minus sign $(9 a)$ refers to the triplet state when the spins are parallel $(S=1)$, the state is $E_{a}+E_{b}+C-A$. The ground orthoheliun state $E_{a}+E_{b}+C-A, 2^{3} S_{1}$ has the energy $59.2 \mathrm{eV}$. The energy of state $2 \mathrm{E}_{a}=2 \cdot 54.4$ $=108.8 \mathrm{eV}$ is doubled ionization energy of the helium ion $\mathrm{He}^{+}$.

In deriving the relation (9b), it is taken into account that the integrals (7) and (8) at $a=b$, are equal. The value of $C$ has a positive value, just like $A$, since the main contribution to the integral (8) is given by those regions of integration in which $r_{12}$ is close to zero, i.e. when the coordinates of electrons coincide, or, in other words, when the wave functions of the electrons overlap. The more the electron wave functions overlap, the greater is the exchange energy value.

\section{EXCHANGE INTERACTION OF ELECTRONS IN HELIUM TRANSATOM}

The wave functions of electrons overlap maximally in the case of electron pairing into an

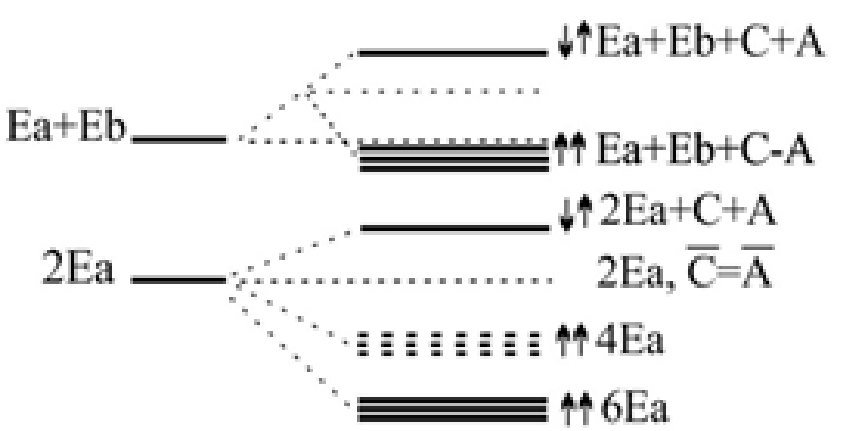

Fig. 1. Scheme of energy levels of helium atom [4] and transhelium under consideration of electron interaction. orthoboson $S=1$. Such pairing of two electrons occurs when the atom is in a strong magnetic field [1]. The motion of two electrons in the orthoboson is rigidly correlated. The electron spins parallel to each other $S=1$ and both electrons are in the same energy state $a=b, E_{a}(1)=E_{a}(2)$. It was shown in paper [1] that the pairing of electrons with parallel spins in a strong magnetic field becomes possible due to, first, exchange interaction of electrons having the character of attraction, and, secondly, to the appearance of electron oscillations near their orbitals [6]. Owing to such oscillations, an additional degree of freedom of motion appears for electrons. A new degree of freedom of movement of electrons generates for them a new quantum number $n_{b}$. Owing to the exchange interaction, the two electrons obtain correlated oscillations. The quantum numbers of oscillations of paired electrons are equal to each other by modulus, but they are opposite in sign $n_{b}^{1}=-n_{b}^{2}, n_{b}=1$, $2,3, \ldots$ Therefore, the Pauli principle for them is satisfied. It is noteworthy that the oscillatory quantum numbers are determined for paired electrons only for each other. The frequency of oscillations $\omega_{\mathrm{B}}$ is related to the frequency of electron rotation on the orbital $\omega_{0}=E_{0} / \hbar$ and the precession frequency of its orbital momentum $\omega_{\ell}$ by the following relation [6-7]:

$\omega_{B}=n_{b} \sqrt{\omega_{0}^{2}+\omega_{\ell}^{2}}$.

The electron oscillations occur both in the longitudinal and transverse magnetic field $B$ directions. As electrons in a pair oscillate in counterphase $n_{b}^{1}=-n_{b}^{2}$, such a motion allows two electrons in equal energy states to be in nonintersecting spatial regions. The trajectories of two moving paired electrons can be represented as closed spirals, which are enclosed into each other and located on the toroidal surface [1].

The oscillatory motion of electrons can be regarded as independent of their orbital motion around the nucleus. Therefore, the wave function of the spatial motion of paired electrons can be represented as their product $\left\{\Psi_{a}(1) \Psi_{a}(2)+\Psi_{a}(2)\right.$ $\left.\Psi_{a}(1)\right\} \times\left\{\Psi_{a}[1,2]-\Psi_{a}[2,1]\right\}$, where $\left\{\Psi_{a}[1,2]-\right.$ $\left.\Psi_{a}[2,1]\right\}-$ is the antisymmetric wave function of 
the oscillatory motion of paired electrons, and $\left\{\Psi_{a}(1) \Psi_{a}(2)+\Psi_{a}(2) \Psi_{a}(1)\right\}$ is the symmetric wave function of the orbital motion electrons around the nucleus. It is obvious that $\Psi_{a}(1) \Psi_{a}(2)=\Psi_{a}(2)$ $\Psi_{a}(1)$. The function $\Psi_{a}[1,2]$ differs from $\Psi_{a}[2,1]$ by oscillation direction of paired electrons, i.e. by motion along the toroidal surface clockwise or counterclockwise. As a result, the total wave function of two correlated electrons with allowance for the symmetric spin part can be written as follows:

$\Psi_{a}(1) \Psi_{a}(2) \times\left\{\Psi_{a}[1,2]-\Psi_{a}[2,1]\right\} \times\left[S^{+}(1) S^{+}(2)\right]$; $\Psi_{a}(1) \Psi_{a}(2) \times\left\{\Psi_{a}[1,2]-\Psi_{a}[2,1]\right\} \times\left[S^{-}(1) S^{-}(2)\right]:$ $\Psi_{a}(1) \Psi_{a}(2) \times\left\{\Psi_{a}[1,2]-\Psi_{a}[2,1]\right\} \times\left[S^{+}(1) S^{-}(2)+S^{+}(2) S^{-}(1)\right]$.

We apply the procedures (5), (6) to the full wave function and introduce the notations $\bar{C}$ and $\bar{A}$

$$
\begin{aligned}
& \bar{C}=\int\left|\psi_{a}(1)\right|^{2}\left|\psi_{a}(2)\right|^{2}\left\{\left|\psi_{a}[1,2]\right|^{2}+\left|\psi_{a}[2,1]\right|^{2}\right\} \times \\
& \times\left[k \cdot e^{2} / r_{1,2}\right] d V_{1} d V_{2}, \\
& \bar{A}=\int\left|\psi_{a}(1)\right|^{2}\left|\psi_{a}(2)\right|^{2}\left\{\psi_{a}^{*}[1,2] \psi_{a}[2,1]+\psi_{a}[1,2] \psi_{a}^{*}[2,1]\right\} \times \\
& \times\left[k \cdot e^{2} / r_{1,2}\right] d V_{1} d V_{2} .
\end{aligned}
$$

The correction to energy $\Delta E_{(1)}$ is written as:

$$
\Delta E_{(1)}=\bar{C}-\bar{A} \text {. }
$$

It is obvious that $\left|\psi_{a}[1,2]\right|^{2}=\left|\psi_{a}[2,1]\right|^{2}$. We represent the wave function of oscillating paired electrons $\Psi_{a}[1,2]$ as the product of wave functions of each individual electron $\Psi_{a}[1,2]=\Psi_{a}[1] \Psi_{a}[2]$. Then $\psi_{a}^{*}[1,2] \psi_{a}[2,1]=\psi_{a}[1,2] \psi_{a}^{*}[2,1]$ and they are equal to $\left|\psi_{a}[1,2]\right|^{2}$. It is clear that integrals (11) and (12) are equal, i.e. $\bar{C}=\bar{A}$. Consequently, the exchange energy between electrons in orthoboson, in the first order of perturbation theory, completely compensates the energy of their Coulomb repulsion and $\Delta E_{(1)}=0$ (13), the state $2 E_{a}$ (Fig. 1).

Thus, the physical interpretation of exchange energy, as the interaction energy according to the Coulomb law of two electrons, each of which is simultaneously in different states $\Psi_{a}$ and $\Psi_{b}$, leads to the conclusion: when the states of two electrons completely coincide $\Psi_{a} \equiv \Psi_{b}$, i.e. one electron becomes identical with the second electron, eventually identical to itself, then the electrons cease to interact. In our case, the situation is saved by the fact that, although the electrons are in the same energy state, $H \Psi_{a}=E_{a} \Psi_{a}=E_{a} \Psi_{b}=H \Psi_{b}$, but they are located in nonintersecting spatial regions. Therefore, the electrons continue to interact with each other.

The energy of the oscillatory motion of an electron according to (10) is equal to $E_{B}=\hbar \omega_{B}$ $=\hbar \cdot n_{b} \cdot \sqrt{\omega_{0}^{2}+\omega_{\ell}^{2}} \cdot$. The orbital momentum in $S$-state is equal to zero $\omega_{\ell}=0$, and at $n_{b}=1, E_{B}=$ $\hbar \omega_{0}$. In this state, the energy of electron oscillation coincides with the energy of its orbital movement $E_{B}=E_{a}$. Thus, the energy of electron level of paired electrons becomes two times lower than that of the level $2 \mathrm{E}_{a}\left(108.8 \mathrm{eV}\right.$ ), the state $4 \mathrm{E}_{a}$ (Fig. 1). At this level, the frequencies of the orbital and oscillatory motion of electrons change, which will entail a further lowering of electron levels. Representing the iteration in the form of geometric progression with the denominator $1 / 2$, we find that the level of paired electrons will drop further by the value $2 E_{a}$. Thus, the energy of paired electrons in the helium transatom is $6 \mathrm{E}_{a}=$ $3 \cdot 108.8=326.4 \mathrm{eV}$ or $163.2 \mathrm{eV}$ for each electron (Fig. 1).

According to the formula for electron binding energy [8]

$E_{e}=k \cdot Z \cdot e^{2} / 2 \mathrm{R}_{\mathrm{THe}}$

the radius of transhelium atom will decrease threefold in comparison with the state $2 \mathrm{E}_{a}=108.8$ $\mathrm{eV}$ and will be equal to $R_{\mathrm{THe}}=8.83 \cdot 10^{-12} \mathrm{~m}$. The magnetic induction vector, created by the magnetic moments of electrons $\mu_{\mathrm{e}}$, in the center of atom, is equal to $B_{0}^{H e}=3.46 \cdot 10^{4} \mathrm{~T}$.

Let us underline that the energy of the oscillatory motion of electrons was not taken into account in [2] in calculating the magnetic fields of the transatoms. It is necessary to make corrections to the energy of electron oscillations and the radius of their orbitals (14) by three times and, accordingly, and for the increase of magnetic induction vector at the center of transatoms by 27 times. This is true for light elements with $Z \leq 16$. For heavier transatoms, there is a restriction on the reduction in the orbital radius of orthobosons, which is related to the minimum permissible distance $a_{\mathrm{Z}}$ between neighboring electrons located on the same orbital, and is equal 
to $a_{\mathrm{Z}} \sim 10^{-12} \mathrm{~m}$. The values of magnetic induction vector for nuclei $Z>16$ will then increase, but no longer by 27 times. Atomic nuclei in ultrastrong fields of transatoms are transformed into transnuclei $[1,2]$.

\section{EXCHANGE INTERACTION OF ELECTRONS IN HYDROGEN MOLECULE}

Unlike helium in a hydrogen molecule, electrons interact not only with each other, but with neighboring nuclei - protons. The ground state of two noninteracting hydrogen atoms $\Psi_{a}(1)=\Psi_{100}\left(r_{1}\right)$ and $\Psi_{b}(2)=\Psi_{100}\left(r_{2}\right)$ are taken as nonperturbed states, and their combination (1a) and (1b) as nonperturbed functions. For a hydrogen molecule, the perturbation energy is equal to [4]:

$$
V^{\prime}=k \cdot\left(\frac{e^{2}}{R}+\frac{e^{2}}{r_{12}}-\frac{e^{2}}{r_{1 b}}-\frac{e^{2}}{r_{2 a}}\right)
$$

for nonperturbed wave function $\Psi_{a}(1) \Psi_{b}(2)$

$$
V^{\prime \prime}=k \cdot\left(\frac{e^{2}}{R}+\frac{e^{2}}{r_{12}}-\frac{e^{2}}{r_{2 b}}-\frac{e^{2}}{r_{1 a}}\right)
$$

for nonperturbed wave function $\Psi_{a}(2) \Psi_{b}(1)$, where $k \cdot e^{2} / R$ - interaction energy of two protons at distance $R, k \cdot e^{2} / r_{1,2}$ - interaction energy between electrons, $-k \cdot e^{2} / r_{1 \mathrm{~b}}$-interaction energy of electron 1 with proton $b,-k \cdot e^{2} / r_{2 a}$ - interaction energy of electron 2 with proton $a$. Interaction energy of electron 1 with proton $a-k \cdot e^{2} / r_{1 a}$ and interaction energy of electron 2 with proton $b-k \cdot e^{2} / r_{2 \mathrm{~b}}$ are taken into account in nonperturbed functions.

We consider the interaction energy as first correction to system energy and write down:

$$
\Delta E(R)=\frac{\int \hat{V} \psi^{*}(1,2) \psi(1,2) d V_{1} d V_{2}}{\int \psi^{*}(1,2) \psi(1,2) d V_{1} d V_{2}} .
$$

Taking into account (15a) and (15b), the numerator is written as follows:

$$
\begin{aligned}
& \int \hat{V} \psi^{*}(1,2) \psi(1,2) d V_{1} d V_{2}= \\
& =\int V^{\prime} \psi_{a}^{2}(1) \psi_{b}^{2}(2) d V_{1} d V_{2}+ \\
& +\int V^{\prime \prime} \psi_{a}^{2}(2) \psi_{b}^{2}(1) d V_{1} d V_{2} \pm \\
& \pm 2 \int \hat{V} \psi_{a}(1) \psi_{b}(2) \psi_{a}(2) \psi_{b}(1) d V_{1} d V_{2} .
\end{aligned}
$$

For the last, mixed integral term, its values are equal for $V^{\prime}$ and $V^{\prime \prime}$.

If we introduce designations $\vec{C}, \vec{A}$ and $\vec{S}$ :

$$
\begin{aligned}
& \vec{C}=k \cdot e^{2} \int\left(\frac{1}{R}+\frac{1}{r_{12}}-\frac{1}{r_{1 b}}-\frac{1}{r_{2 a}}\right) \psi_{a}^{2}(1) \psi_{b}^{2}(2) d V_{1} d V_{2}, \\
& \vec{A}=k \cdot e^{2} \int\left(\frac{1}{R}+\frac{1}{r_{12}}-\frac{1}{r_{1 b}}-\frac{1}{r_{2 a}}\right) \times \\
& \times \psi_{a}(1) \psi_{b}(1) \psi_{a}(2) \psi_{b}(2) d V_{1} d V_{2}, \\
& \vec{S}=\int \psi_{a}(1) \psi_{b}(2) \psi_{a}(2) \psi_{b}(1) d V_{1} d V_{2},
\end{aligned}
$$

then the corrections to energy can be presented in the form:

$$
E^{+}(R)=(\vec{C}+\vec{A}) /(1+\vec{S})
$$

for symmetric coordinate function and, correspondingly, for antisymmetric spin function and

$$
E^{-}(R)=(\vec{C}-\vec{A}) /(1-\vec{S})
$$

for antisymmetric coordinate function and for symmetric spin function.

At distances $R$ of the order of Bohr radius of electron $R_{H}$, the overlap of electron wave functions is significant and therefore their exchange density is large. The magnitude of the exchange integral $\vec{A}$ at these distances is determined by the attraction of electrons to protons, i.e. by the terms $-k \cdot e^{2} / r_{1 \mathrm{~b}}$ and $-k \cdot e^{2} / r_{2 \mathrm{a}}$, thereby making the integral value $\vec{A}$ negative. At short distances $R$, the interaction between hydrogen atoms is determined by the repulsion of protons, both in case $E^{+}(R)$ and in case $E^{-}(R)$. At nuclear distances between protons, a hydrogen molecule is converted into an atom of "helium".

At medium distances, hydrogen atoms with parallel electron spins repel $E^{-}(R)$, and those with antiparallel spins attract $E^{+}(R)$. The changes in potential energies $E^{+}(R)$ and $E^{-}(R)$ are shown in Fig. $2 a$ depending on the distance between
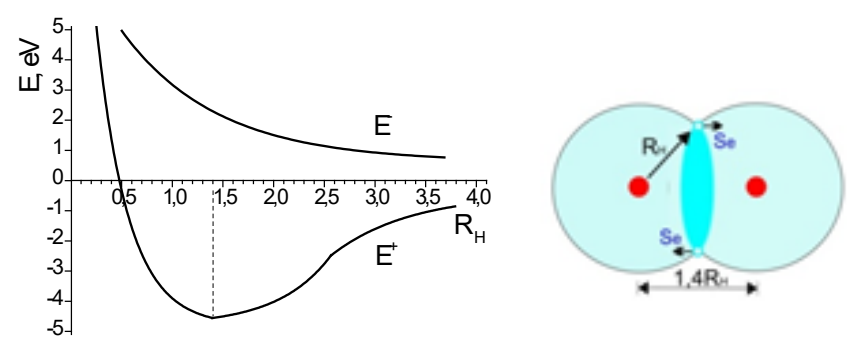

Fig. 2. Change of potential energy $\mathrm{E}^{-}$and $\mathrm{E}^{+}$and bydrogen molecule. 
the protons. In case $E^{+}(R)$, a hydrogen molecule is formed in which the total pair of electrons equally belongs to both hydrogen atoms (Fig. 2b), $S_{\mathrm{e}}$ - electron spins. Experimental data show that the equilibrium distance between protons in the hydrogen molecule is $1.4 \mathrm{R}_{\mathrm{H}}$, where $R_{\mathrm{H}}=$ $5.3 \cdot 10^{-11} \mathrm{~m}$ is the Bohr radius of the electron, and its dissociation energy is $4.5 \mathrm{eV}(35000 \mathrm{~K})$. Conditionally, we can assume that the electrons located between the protons in the hydrogen molecule are also located at a distance of $1.4 \mathrm{R}_{\mathrm{H}}$ due to their Coulomb repulsion (Fig. 2b).

\section{INTERACTION OF HYDROGEN ATOMS IN STRONG MAGNETIC FIELD. FORMATION OF HYDROGEN TRANSMOLECULE}

We consider the interaction of two hydrogen atoms in a strong magnetic field $\mathbf{B}$ (in the range 20-100 T). In a magnetic field, the ground electronic level in a hydrogen atom splits into two levels with multidirectional electron spins. The splitting of the ground state is $\Delta E=2 \mu \cdot B$, which corresponds to the values $(0.23-1.2) \cdot 10^{-2} \mathrm{eV}$, where $\mu_{\mathrm{e}}=9.29 \cdot 10^{-24} \mathrm{~J} / \mathrm{T}=5.79 \cdot 10^{-5} \mathrm{eV} / \mathrm{T}$ is the magnetic moment of an electron. These splitting values are less than the thermal energy of 0.025 $\mathrm{eV}$ at a temperature of $300 \mathrm{~K}$. Therefore, at temperatures above $300 \mathrm{~K}$, the split levels will be populated with electrons evenly, half of hydrogen atoms will have an electron spin directed parallel to the magnetic field, and the other half of hydrogen atoms will have an electron spin directed against the magnetic field. Hydrogen atoms with multidirectional electron spins will attract each other and atoms with parallel spins will repel.

In addition, atomic electrons under the action of other charges will oscillate, in a strong magnetic field, near their orbitals $[1,6]$.

The magnetic moments of electrons $\mu_{\mathrm{e}}$, oriented both along the field and against the magnetic field $\mathbf{B}$, create intrinsic magnetic fields $\mathbf{B}_{\mathrm{s}}$ around the atoms, which are equal to [9]:

$$
\mathbf{B}_{S}=\mu_{0} \frac{3 \mathbf{n}\left(\boldsymbol{\mu}_{e} \cdot \mathbf{n}\right)-\boldsymbol{\mu}_{e}}{r^{3}}
$$

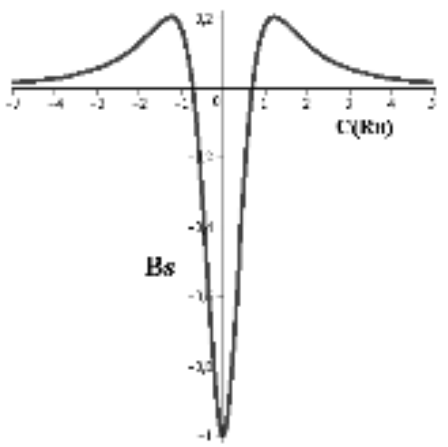

Fig. 3. Dependence of magnetic induction vector value $\mathbf{B}_{\mathrm{s}}$ along axis $C\left(\mathrm{R}_{\mathrm{H}}\right)$.

where $\mu_{0}=1.26 \cdot 10^{-6} \mathrm{H} / \mathrm{m}$ is magnetic constant; $r$ is the distance from electron to the point at which the $\mathbf{B}_{\mathrm{s}}$ field is calculated; $\mathbf{n}$ is the unit vector in the $r$-direction. Fig. 3 shows the calculated dependence of the magnitude of the magnetic induction vector $\mathbf{B}_{\mathbf{s}}$ in relative units along the $C\left(R_{H}\right)$ axis passing through the atom's centre and parallel to external magnetic field $\mathbf{B}[2]$. The $C\left(R_{H}\right)$ axis is given in units of the Bohr electron radius $R_{H}$. Negative values of $\mathbf{B}_{\mathbf{s}}$ mean that the magnetic field in central region of the atom is directed opposite to the direction of the magnetic moment of electron. The second, positive maximum is at a distance of $1.22 \mathrm{R}_{\mathrm{H}}$ from the proton, and at a distance of $0.71 R_{\mathrm{H}}$ the magnetic field is equal to zero.

The calculation showed that magnetic fields of the hydrogen atom have values at the center $B_{S}^{0}=79 \mathrm{~T}$, and at a distance of $1.2 \cdot \mathrm{R}_{\mathrm{H}}$ from the center along the $C\left(R_{\mathrm{H}}\right)$ axis, at a positive maximum $B_{S}^{R_{H}}=16 T$ [10] (Fig. 3). The magnetic interaction between hydrogen atoms $\mu_{\mathrm{e}} B_{S}^{R}$ has the character of attraction for atoms with parallel spins of electrons and it has the character of repulsion with multidirectional electron spins. $B_{S}^{R}$ is magnetic field created by the magnetic moment of the electron $\mu_{e}$ of the first hydrogen atom on electron orbital $\left(R_{H}\right)$ of another hydrogen atom. Fig. 4a-d shows the sum of magnetic fields along the $C\left(R_{H}\right)$ axis from two co-axial hydrogen atoms with parallel electron spins, depending on the distance between the protons $R$. At distances $R \leq 0.7 \cdot R_{H}$ (Fig. 4d), the protons are in the same "magnetic well", created by two electrons. The energy of magnetic interaction between the electrons of 


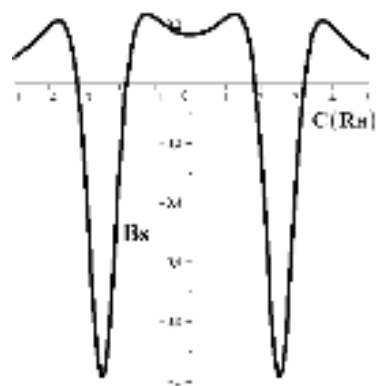

a) $\mathrm{R}=5 \mathrm{R}_{\mathrm{H}}$

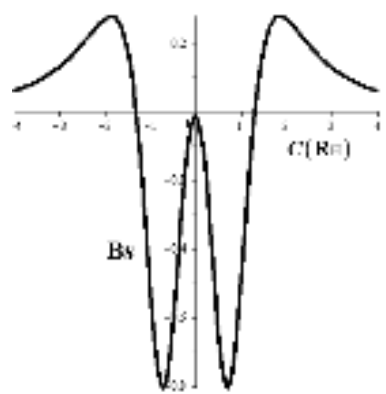

c) $\mathrm{R}=1.4 \mathrm{R}_{\mathrm{H}}$

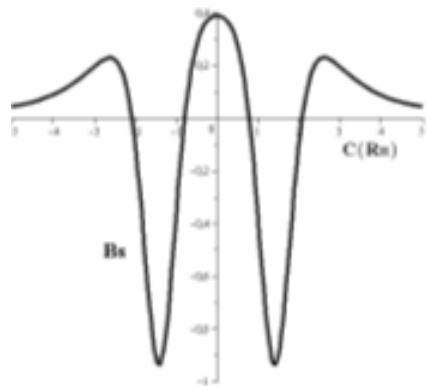

b) $R=2.4 R_{H}$

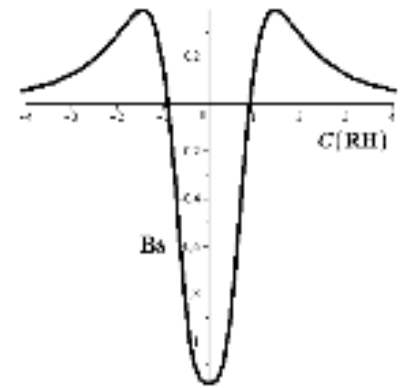

d) $\mathrm{R}=0.7 \mathrm{R}_{\mathrm{H}}$
Fig. 4. Magnetic field sums along axis $C\left(R_{H}\right)$ from two bydrogen atoms as function of distance between nuclei $\mathrm{R}$.

hydrogen atoms is $E_{\mu}=2 \mu_{\mathrm{e}} B_{S}^{R}$. It is smaller than their exchange energy and smaller than the energy between the split levels.

When the hydrogen atoms come closer to each other according to their relative velocity $W(t)$, the magnetic field $B_{S}^{R}$ begins to depend on time $B_{S}^{R}(W(t))$ (Fig. 4). Consequently, the change of spin direction of the electron in the hydrogen atom under the action of the field $B_{S}^{R}(t)$, i.e. electron transition from the upper split level to the lower level becomes possible. This transition is accompanied by energy release. The reverse transition is forbidden, since it occurs with energy absorption, which must be taken from somewhere.

We suppose that two hydrogen atoms approach each other due to thermal motion along an axis parallel to the direction of a strong magnetic field B. And let the electrons be on the split levels $E_{a}(1)$ and $E_{b}(2)$ with oppositely directed spins. The atoms will attract each other due to exchange interaction.

With a decrease in the distance between hydrogen atoms under the action of Coulomb interaction, the electrons in the atoms begin to oscillate near their orbitals. In this case, the oscillations of electron in one atom will be synchronized with the oscillations of the electron in the second atom. Such synchronization, associated with a decrease in the Coulomb interaction between electrons, leads to the fact that the quantum numbers of their oscillations will be equal to each other $n_{b}^{1}=n_{b}^{2}$. In the end, their quantum numbers will become equal to one $n_{b}^{1}=n_{b}^{2}$ $=1$. Since electrons are at different levels $\omega_{01} \neq \omega_{02}$, the frequencies of their oscillations will differ from each other $\omega_{\mathrm{B} 1} \neq \omega_{\mathrm{B} 2}(10)$. In this case, the moments of the pulses of two electrons are not defined for each other and their oscillatory wave function does not have a certain symmetry [1].

In an time-varying magnetic field $B_{S}^{R}(\mathrm{t})$, an electron can transfer from the upper split level to the lower level. Such a transition will cause the electrons to have parallel spins $S=$ 1 ; they will be in the same energy states $E_{a}(1)$ $=E_{a}(2), \omega_{01}=\omega_{02}$, hence, $\omega_{\mathrm{B} 1}=\omega_{\mathrm{B} 2}$ and the quantum numbers of electron oscillations will be equal in modulus and opposite in sign $n_{b}^{1}=-n_{b}^{2}$. Consequently, the total wave function of the electron pair will remain antisymmetric, since it consists of the product of a symmetric orbital coordinate function, an antisymmetric oscillatory coordinate function (independent of the orbital function), and a symmetric spin function. Each electron in the pair acquires an additional quantum number: one is $n_{b}^{1}$ and the other is $-n_{b}^{2} \quad(1$ and -1$)$. Consequently, the electrons remain antisymmetric. Further approaching will cause pairing of electrons and the formation of an orthoboson. Like in transhelium (chapter 3), exchange interaction $\bar{A}$ of paired electrons is completely compensated by their Coulomb repulsion $\bar{C}$. For this reason, an orthoboson transfers from the energy state with radius $R_{H}$ to a low-lying state with a radius $R_{\mathrm{T}}$ (Fig. 5a). By analogy with helium transatom,

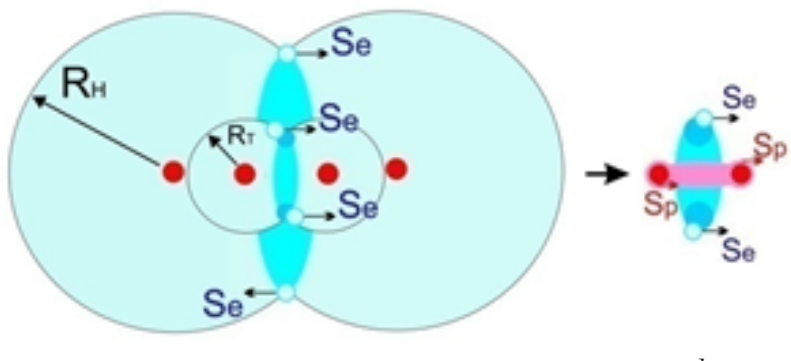

Fig. 5. Transfer of bydrogen molecule into bydrogen transmolecule. 
the radius $R_{T}\left(1.76 \cdot 10^{-11} \mathrm{~m}\right)$ can be three times smaller than $R_{H}$. Accordingly, the distance between electrons will be $2.46 \cdot 10^{-11} \mathrm{~m}\left(1.4 \cdot \mathrm{R}_{\mathrm{T}}\right)$, and the binding energy of each electron is $\sim 116$ $\mathrm{eV}$ (14). Approaching of electrons will lead to a corresponding approaching of protons. Thus, a hydrogen transmolecule " $\mathrm{H}_{2}$ " is formed in this way (Fig. 5b).

The same effect can be caused by a collision of atoms with parallel spins of electrons. But for this purpose, the kinetic energy of atoms must be greater than the energy of their repulsion due to the exchange interaction of electrons, until the appearance of correlated electron oscillations.

The process of formation of hydrogen transmolecule can occur in a different way. First, a hydrogen transatom " $H$ " is formed due to the electron oscillations available in the hydrogen atom. At that, a photon with an energy of 2.13.6 $=27.2 \mathrm{eV}$ is emitted. Next, two transatoms of hydrogen create a hydrogen transmolecule.

In strong and ultrastrong variable magnetic fields, the hydrogen molecule itself can become a hydrogen transmolecule.

\section{NUCLEAR REACTIONS WITHOUT COULOMB BARRIER}

Protons and other transnuclei in the centers of transatoms and transmolecules [1-3] are located in an electromagnetic well. Electrostatic forces of the toroid of electron Bose condensate create at its center a potential well with a minimum energy of electron interaction with transnuclei. The deviation of transnuclei from the center of the toroid causes an increase of Coulomb forces, which tend to return the transnuclei. In the center of the toroid, the magnetic well has the maximum value of the magnetic induction vector $B_{S}^{0}$. Consequently, the energy of interaction with the magnetic moments of the transnuclei will be maximal in the center of the toroid. Magnetic interaction attracts the transnuclei to the center. Since the Bose electrons in the transatom are distributed on the surface of the toroid, which cross-sectional dimensions are comparable to the orbital radius of orthobosons, then the bottom of, both electrostatic and magnetic potential wells has a parabolic appearance as shown

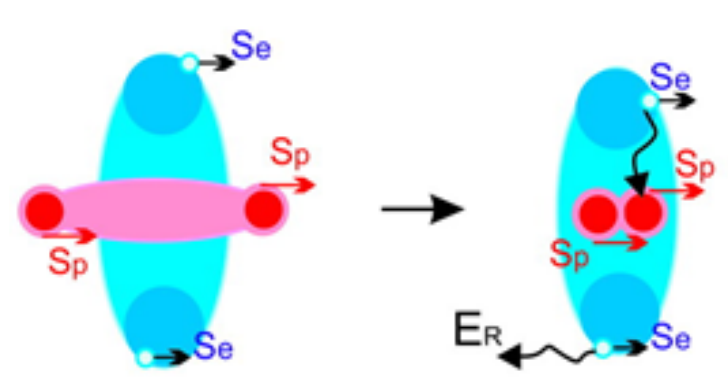

Fig. 6. Transformation of bydrogen transmolecule into "belium" transmolecule.

in Fig. $4 d$. The radius $R_{z}$ of the orbital of electron $\mathrm{R}_{\mathrm{z}} \cdot m_{\mathrm{e}} \cdot V_{\mathrm{e}}=\hbar$ for the main quantum number $n=1$, is equal to its wavelength $\lambda=\hbar / m_{\mathrm{e}} V_{\mathrm{e}}$.

In the hydrogen atom, the proton rotates together with electron around their common center of mass. The electron oscillations that arise in a strong magnetic field cause corresponding oscillations in the motion of the proton near its orbital. Two protons of a hydrogen transomolecule in an electrostatic potential well will rotate around the center of the transmolecule and oscillate due to the magnetic field produced by electron orthoboson. When the distance between paired electrons in a hydrogen transmolecule is $2.46 \cdot 10^{-11} \mathrm{~m}$, the magnetic induction vector at the center of the "magnetic well" will have a value of $\sim 10^{4} \mathrm{~T}$. The protons in such a ultrastrong and inhomogeneous magnetic field will have parallel spins $\left(S_{p}=1 / 2\right)$. The protons, like electrons in the hydrogen transmolecule form a bound state - an orthoboson $S=1$ due to the intrinsic exchange interaction and intrinsic correlated oscillations (Fig. 6a, Chapter 3). Just as in the case of electrons, the exchange interaction of protons in the first order of perturbation theory completely compensates their Coulomb repulsion. This will lead to approaching of protons up to nuclear distances, formation of transmolecule "heliumpp" (Fig. 6b) and to the beginning of the nonCoulomb nuclear reaction.

ISOTOPES OF HYDROGEN. If the lifetime of the "helium-pp" transmolecule is $\tau_{\mathrm{pp}}>>2 \cdot 10^{-18}$ $c(\mathrm{~h} /(2 \cdot 163 \mathrm{eV}))$, then the reaction $p+p \rightarrow d+$ $v_{e}+e^{+}+0.42 \mathrm{MeV}$ should be suppressed. Then the most probable fusion reaction of two protons with the formation of a deuteron $d$ is a reaction involving coupled electrons, each of which is 
already located at a level with an energy of 163.2 $\mathrm{eV}\left(\mathrm{R}_{\mathrm{THe}}=8.84 \cdot 10^{-12} \mathrm{~m}\right)$ and with magnetic induction vector at the center of the "helium" transmolecule $B_{0}^{\mathrm{He}}=3.5 \cdot 10^{4} \mathrm{~T}$ :

$p+p+2 e^{-} \rightarrow d+v_{e}+e^{-}+1.44 \mathrm{MeV}$.

The reaction is recorded with the participation of two coupled electrons, since the energy released in it is more convenient to be realized due to the recoil energy $E_{\mathrm{R}}$ of the second correlated electron (Fig. 6b). For this reason, the electron spectrum can be similar to the beta decay spectrum.

Obviously, the identical atoms of other hydrogen isotopes: deuterium $D$ and tritium $T$, can also form "helium-dd" and "helium-tt" transmolecules in a strong magnetic field. And the deuteron nuclei with spin 1 and tritons $t$ with spin $1 / 2$ can enter the non-Coulomb nuclear fusion reactions:

$$
\begin{aligned}
& d+d \rightarrow t+p+4.03 \mathrm{Mэ} \mathrm{B}, \\
& d+d \rightarrow{ }^{3} \mathrm{He}+n+3.26 \mathrm{MэB}, \\
& t+t \rightarrow{ }^{4} \mathrm{He}+2 n+11.3 \mathrm{MэB}, \\
& d+d+2 e^{-} \rightarrow{ }^{4} \mathrm{He}+2 e^{-}+23.85 \mathrm{MэB} .
\end{aligned}
$$

The last reaction with electron participation, if $\tau_{\mathrm{dd}}>>2 \cdot 10^{-18} \mathrm{c}$, can have a substantial yield comparable with reactions $(19,20)$, but not with reaction $d+d \rightarrow{ }^{4} \mathrm{He}+\gamma+23.85 \mathrm{MeV}$, which has a yield $\sim 10^{-7}$. If $\tau_{\mathrm{tt}}>>2 \cdot 10^{-18} \mathrm{c}$, the following reaction becomes possible:

$t+t+2 e \rightarrow 2 e^{-}+12.3 \mathrm{MeV}+{ }^{6} \mathrm{He}\left(\beta, T_{1 / 2}=0.8 c\right)$

$\rightarrow{ }^{6} \mathrm{Li}+\mathrm{e}^{-}+\tilde{v}_{e}+3.5 \mathrm{MeV}$.

ISOTOPES OF HELIUM. Parahelium electron levels in a strong magnetic field are split into two levels with multidirectional electron spins. Cross-exchange interactions between attracting multidirectional spins of electrons, and between repulsive parallel spins of electrons will compensate each other. That is why, in order for the nuclear reaction to begin, the kinetic energy of parahelium colliding atoms must be such that correlated electron oscillations arise on atomic orbitals.

Orthohelium has an atomic spin $S=1$. The radius of orbital $R_{1}$ of the first orthohelium electron is $3.1 \cdot 10^{-11} \mathrm{~m}$, the radius of orbital $R_{2}$ of the second electron is $8.76 \cdot 10^{-11} \mathrm{~m}$. The orthohelium ionization energy is $4.8 \mathrm{eV}$, that of the parahelium is $24.6 \mathrm{eV}$. In a strong magnetic field, the level of the external electron $2^{3} \mathrm{~S}_{1}$ splits into three levels with spin projections $-1,0,+1$.

Just as in case of hydrogen, in orthohelium:

- the split levels will be populated with electrons evenly;

- orthohelium atoms with oppositely directed spins of electrons will be attracted to each other, and atoms with parallel spins will be repelled;

- in the case of attraction of orthohelium atoms, with decreasing distance between them under the influence of Coulomb interaction, internal and external electrons begin to oscillate in them synchronously about their orbitals;

- electron transitions from the split upper levels $(+1,0)$ to the lower level $(-1)$ will occur under the action of an alternating magnetic field $B_{S}^{R}(t)$.

The spins of orthohelium internal and external electrons are always parallel, consequently their interactions, both with electron spins of other orthohelium, and inside the atom, are always synchronous. Therefore, after the creation of electron orthobosons, the external orthoboson descends to the level of internal orthoboson. Thus, the Bose condensate and the beryllium transmolecule "Be" are formed. Consequently, the nuclei will approach and, in the case of ${ }^{3} \mathrm{He}$, the spin of the nucleus $I=1 / 2$, a non-Coulomb nuclear reaction will begin

$$
{ }^{3} \mathrm{He}+{ }^{3} \mathrm{He} \rightarrow{ }^{4} \mathrm{He}+2 p+12.86 \mathrm{MeV} .
$$

The orthohelium atom, in comparison with the hydrogen atom, has a substantial magnetic field. The calculation showed that magnetic fields of orthohelium atom have values in the center $B_{S}^{0}$ $(H e)=410 \mathrm{~T}$, and at a distance of $1.2 \cdot \mathrm{R}_{2}$ from the center along the $C\left(R_{\mathrm{H}}\right)$ axis, at a positive maximum (Fig. 3) $B_{S}^{R_{2}} \sim 70 T$. Therefore, as shown by the experiment [10], non-Coulomb nuclear reactions in the orthohelium can proceed due to intrinsic magnetic fields.

In case of another helium isotope ${ }^{4} \mathrm{He}$, a paired non-Coulomb nuclear reaction is impossible for energy reasons $Q<0$. However, two orthohelium ${ }^{4} \mathrm{He}$ form a stable beryllium transmolecule " $B e$ " (Fig. 7a). The radius of transmolecule " ${ }^{8} e^{\prime \prime}$ is $\mathrm{R}_{\mathrm{Be}}=4.4 \cdot 10^{-12} \mathrm{~m}$, and the magnetic fields: in the center $B_{S}^{0}(B e)=5.4 \cdot 10^{5} T$ and at $1.2 \cdot \mathrm{R}_{\mathrm{Be}}$ from the center $B_{S}^{R}(B e)=1.1 \cdot 10^{5} \mathrm{~T}$. 

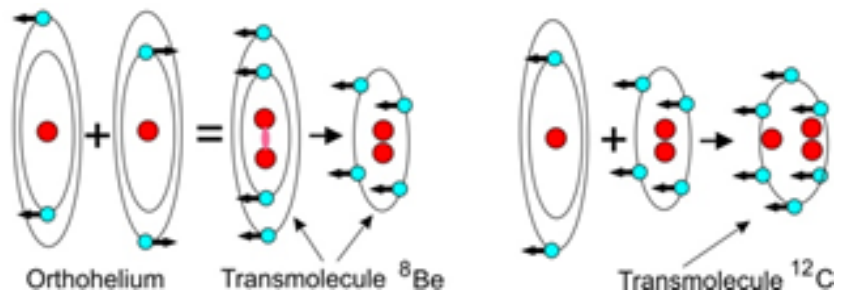

Fig. 7. Formation of transmolecules ${ }^{8} \mathrm{Be}$ and ${ }^{12} \mathrm{C}$.

In the absence of a strong (ultrastrong) magnetic field, in spite of the fact that the ${ }^{4} \mathrm{He}$ nuclei are bosons $I=0$, they can not form a nuclear molecule due to the Coulomb repulsion. The situation changes when two helium nuclei are in a ultrastrong magnetic field. In the magnetic field, due to its interaction with the magnetic moments of protons and neutrons, the nuclear levels in helium are split into $\Delta E_{(p, n)}= \pm \mu_{(p, n)} \cdot B_{S}^{0}, \mu_{\mathrm{p}}$ $=8.79 \cdot 10^{-8} \mathrm{eV} / T, \mu_{\mathrm{n}}=-6.02 \cdot 10^{-8} \mathrm{eV} / T$ (Fig. 8). As proton magnetic moment is parallel to the spin $S_{\mathrm{p}}$, and neutron magnetic moment is antiparallel to the spin $S_{n}$ and it has a negative value, a nonzero mechanical moment $S_{p} \downarrow \downarrow S_{n}$ is created on the outer level of the helium nucleus, which is not compensated by the internal mechanical moment $S_{p} \uparrow \uparrow S_{n}$. This mechanical moment, however small, generates an exchange interaction between the two helium nuclei, which creates the transmolecule "8 $\mathrm{B} e$ ". We remind you that coupled identical charged particles (atomic electrons, protons, atomic nuclei) should be in equal energy states, which requires the same direction and equality of their spins $\uparrow \uparrow$. Absolute values of spins are of no importance. All even-even nuclei with a spin equal to zero $(I=$ 0 ) acquire a mechanical moment $I \neq 0$ in a strong (ultrastrong) magnetic field and cease to be bosons.

The atom of orthohelium, and possibly of parahelium in the ultrastrong alternating magnetic

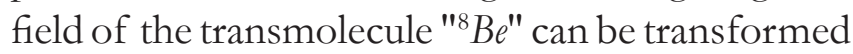

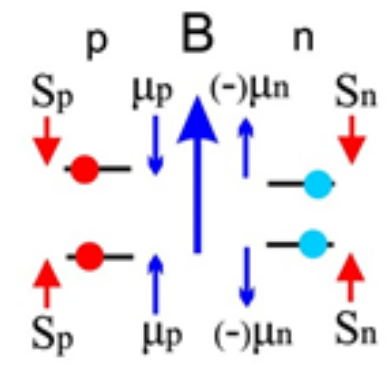

Fig. 8. Appearance of mechanical moment in nucleus ${ }^{4} \mathrm{He}$ in ultrastrong magnetic field. into atoms of the transhelium and create a stable carbon transmolecule ${ }^{12} \mathrm{C}^{\prime \prime}$. It consists of three ${ }^{4} \mathrm{He}$ nuclei: two paired helium nuclei and one unpaired ${ }^{4} \mathrm{He}$ (Fig. $7 \mathrm{~b}$ ). The radius of transmolecule ${ }^{12} \mathrm{C}^{\prime}$ is $R_{C}=3.0 \cdot 10^{-12} \mathrm{~m}$, and magnetic fields: in the center of $B_{0}(C)=2.6 \cdot 10^{6} \mathrm{~T}$ and at a distance of $1.2 \cdot \mathrm{R}_{\mathrm{C}}$ from the center $B_{S}^{R}(C)=5.2 \cdot 10^{5} \mathrm{~T}$. Subsequently, the transmolecules " ${ }^{18} e^{\prime \prime}$ and ${ }^{~}{ }^{12} C^{\prime}$, due to their own ultrastrong magnetic fields, will be attracted to each other, and enter into an exchange interaction with their electron Bose-condensates. This will result in formation of multinuclear transmolecules $n \cdot{ }_{2}^{4} \mathrm{He}$ with helium Bose condensate. The creation of such transmolecules leads to multinuclear reactions, with the emission of protons, neutrons, alpha particles and heavy fragments [10]:

$$
\begin{aligned}
& n \cdot{ }_{2}^{4} \mathrm{He} \rightarrow{ }_{2 n-1}^{4 n-1} \mathrm{~B}+p+Q, \\
& n \cdot{ }_{2}^{4} \mathrm{He} \rightarrow{ }_{2 n}^{4 n-1} B+n+Q, \\
& n \cdot{ }_{2}^{4} \mathrm{He} \rightarrow{ }_{2(n-1)}^{4(n-1)} \mathrm{A}+{ }_{2}^{4} \mathrm{He}+Q,
\end{aligned}
$$

where $Q$ is energy released as a result of reaction.

ELEMENTS WITH $Z>2$. Lithium and boron are odd elements. The atomic nuclei of their stable isotopes have non-zero spins $I\left({ }_{3}^{6} L i\right)=1, I($ $\left.{ }_{3}^{7} L i\right)=3 / 2, I\left({ }_{5}^{10} B\right)=3, I\left({ }_{5}^{11} B\right)=3 / 2$. Therefore, the identical transnuclei of these elements can enter into non-Coulomb nuclear reactions like the isotopes of hydrogen. The atomic nucleus of stable beryllium has a spin $I\left({ }_{4}^{9} B e\right)=3 / 2$, but this is an even element. But since the structure of electron levels ${ }_{4} B e$ has a state with parallel spins of electrons $3^{3} S_{1}$, analogous to the state in the orthohelium $2^{3} S_{1}$, transnuclei ${ }_{4}^{9} \mathrm{Be}$ will enter into the non-Coulomb nuclear reactions.

The electronic states of chemical elements with $Z \geq 6$ have orbital moments different from zero $p, d \ldots(\ell \neq 0)$. Therefore, in a strong magnetic field, due to the intra-atomic electron magnetic resonance, the atoms of these elements will be transformed into transatoms possessing electron Bose condensate [1]. Consequently, after the formation of transmolecules, the identical transnuclei of chemical elements with $Z \geq 6$ will enter into non-Coulomb nuclear reactions.

Transatoms, due to the magnetic and exchange attraction of their electron Bose condensates, form binuclear and multinuclear transmolecules. 
Transmolecules are in the region of a giant inhomogeneous and anisotropic magnetic field (Fig. 3). Consequently, the space in which the transnuclei move in a transmolecule is inhomogeneous and anisotropic. Thus, motion integrals are not conserved in the interaction of the transnuclei: momentum conservation law, angular momentum (spin) conservation law, and energy conservation law are violated. [2]. As a result, the non-radioactive low energy multinuclear reactions (LENR or transmutation reactions) automatically take place in the nuclear transmolecule.

\section{EXPERIMENTS}

Below are described several experiments that find their explanation within the framework of nonCoulomb low energy nuclear reactions. I hope that other numerous experiments will also be explained by the mechanism proposed.

- Vachaev A.V. and Ivanov N.I. performed experiments on elements transmutation in electrical discharge in liquid media [11]. The installation consisted of two tubular electrodes with an internal diameter of 6 to $50 \mathrm{~mm}$, located opposite each to other at a distance of 1-1.5 of their diameter. A specific electrical discharge plasmoid was created between electrodes inside which liquid was moving. The electrodes were located inside the coil, which created a magnetic field. The plasmoid was ignited by a pulsed discharge of additional electrodes located across the jet of liquid, between the tubular electrodes. The plasmoid represented electrically conductive plasma film, which formed a multidimensional figure of the hyperboloid type of revolution with a squeezed diameter of 0.1-0.2 $\mathrm{mm}$. The discharge proceeded almost noiselessly with a minimum release of heat and gas phase. The value of the current through the tubular electrodes varied within 0.1-100 $A$, but in most cases the current was equal to $20-40 A$. The installation could operate, after the formation of a stable plasmoid, autonomously, without an external power source, and it produced electricity itself.

As a result of installation operation, a stable process of transformation of initial material of the medium into new elements and their compounds
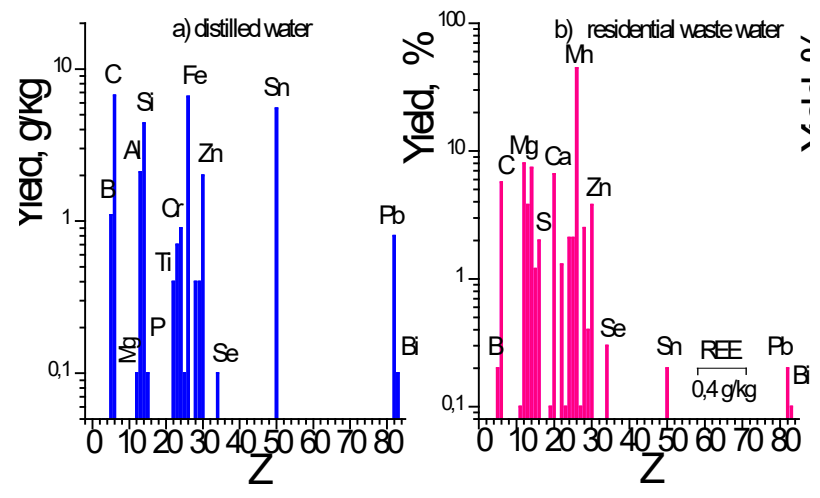

Fig. 9. Element yield at treatment of distilled water and domestic effluents in electrical discharge.

appeared in it, in gaseous, liquid (dissolved in the medium) and solid state. Various liquid media were supplied in experiments to the plant entrance: water (distilled, drinking, river water), water-ore mixtures, effluents of various industries, watercarbon, phenolic and benzene mixtures. All the works were carried out in a mode with maximum yield of solid phase, on the average, up to $300 \mathrm{~g} / \mathrm{l}$. Fig. 9 shows as an example the yields of foreign elements for the treatment of distilled water and domestic waste, REE - rare earth elements.

It is noteworthy that the isotopes of hydrogen and helium were found in the transmutation products: deuterium $(D)$ and tritium $(T),{ }^{3} \mathrm{He}$ и ${ }^{4} H$ e. Table 1 shows the concentrations of $\mathrm{D}_{2} \mathrm{O}$ and $\mathrm{T}_{2} \mathrm{O}$ detected in the installation after treatment with distilled, drinking and river water. Deuterium and tritium were recorded in water after treatment of other mixtures and effluents. For example, after treatment of mixtures of water and various metallic ores, $\mathrm{D}_{2} \mathrm{O}+\mathrm{T}_{2} \mathrm{O}$ were found in concentrations in the range $(0.11-0.18) \%$. After treatment of water-phenolic and waterbenzene effluents, $\mathrm{D}_{2} \mathrm{O}$ molecules were recorded in the range $(0.018-0.60) \%$, and $\mathrm{T}_{2} \mathrm{O}$ in the range $\left(10^{-3}-10^{-4}\right) \%$.

The investigation of the carbon phase, after the treatment of phenolic effluents, showed that up to $50 \%$ of the total mass was

Table 1.

Deuterium and tritium content in water after treatment of various waters

\begin{tabular}{|c|c|c|c|}
\hline & Distilled & Drinking & River \\
\hline$D_{2} \mathrm{O}, \mathrm{mg \Lambda}$ & 0.06 & 0.112 & 0.05 \\
\hline$T_{2} \mathrm{O}, \mathrm{mg} / \mathrm{l}$ & 0.05 & 0.001 & 0.00 \\
\hline
\end{tabular}


graphitized and had the appearance of sooty band-layered dense structures containing $\mathrm{H}_{2}$ up to $14-16 \%,{ }^{3} \mathrm{He}$ up to $0.1 \%$ and ${ }^{4} \mathrm{He}$ up to $0.1 \%$. Similar results were obtained when analyzing the solid phase during the treatment of benzene effluents. The atomic-absorption analysis showed that the liquid phase after treatment of both phenolic and benzene effluents consisted mainly of hydrates of the type $\mathrm{Me} \cdot n \mathrm{H}_{2} \mathrm{O}$, gas hydrates of the form $\mathrm{H}_{2} \cdot 3 \mathrm{H}_{2} \mathrm{O}, \mathrm{D}_{2} \cdot 6 \mathrm{H}_{2} \mathrm{O}$ and traces of $\mathrm{T}_{2} \cdot 18 \mathrm{H}_{2} \mathrm{O}$. In all experiments, the formation of compounds with helium isotopes ${ }^{3} \mathrm{He} \cdot 4 \mathrm{H}_{2} \mathrm{O}$ up to $0.19 \%,{ }^{4} \mathrm{He} \cdot 8 \mathrm{H}_{2} \mathrm{O}$ up to $0.84 \%$ and ${ }^{5} \mathrm{He} \cdot 16 \mathrm{H}_{2} \mathrm{O}$ (?) up to $0.05 \%$ was noted. The liquid phase, in comparison with solid phase had 2.5 -fold increased concentration of helium and 1.8-fold increased concentration of hydrogen gas hydrates. Also, black oil suspensions were treated in a ratio of water equal to $1 / 10$. Analyzes of solid and liquid phases after treatment showed approximately the same results as those obtained in the treatment of phenolic and benzene effluents. The exception was the content of hydrogen and helium isotope gas hydrates, which turned out to be the following on the average: $\mathrm{H}-0.06 \%, \mathrm{H}_{2}-$ up to $6.1 \%, D-0.24 \%, T-0.07 \%,{ }^{4} H-0.001 \%$ (?), ${ }^{3} \mathrm{He}-0.1 \%,{ }^{4} \mathrm{He}-0.5 \%$.

It should be assumed that local regions "capsules" with a strong magnetic field inside the plasmoid are formed [3]. Low energy multinuclear reactions occur in these "capsules". Since ${ }^{3} \mathrm{He}$ and ${ }^{4} \mathrm{He}$ are found in large quantities in reaction products, it is likely that "capsules" are stable formations and within them there are either multiproton reactions or a cascade of $N$ reactions $N_{1} \cdot(p$ $\left.+p+e \rightarrow v_{e}+d\right) \rightarrow N_{2} \cdot(d+d) \rightarrow t,{ }^{3} \mathrm{He},{ }^{4} \mathrm{He} \rightarrow$ $\mathrm{N}_{3} \cdot(t+t) ; \mathrm{N}_{4}\left({ }^{3} \mathrm{He}+{ }^{3} \mathrm{He} e\right) ; \mathrm{N}_{5}\left(n^{\cdot}{ }^{4} \mathrm{He} e\right) \ldots$

- Many hours of irradiation by bremsstrahlung gamma quanta with a maximum energy of $E_{\max }=10 \mathrm{MeV}$ and $E_{\max }=23 \mathrm{MeV}$ of metal samples in an atmosphere of gaseous deuterium, hydrogen and helium at pressures of hundreds to thousands bar were performed in numerous works executed by A.Yu. Didyk, R. Wisniewski et al. at the FLNR of JINR [12-16]. At such pressures, the density of gas atoms is comparable to the density of atoms in solids and liquids. Subsequently the term condensed gas is used. The same investigations were carried out in chambers with pure condensed gases: hydrogen, deuterium, helium and xenon [14-16] at their irradiation by gamma rays with $E_{\max }=10 \mathrm{MeV}$. In all experiments, after the end of the irradiation in the reaction chambers, solid structures containing "foreign" chemical elements that were absent in chambers prior to the beginning of irradiation were found. The range of "foreign" elements extended from hydrogen to bismuth. Isotopes of radioactive elements were detected. Fig. 10 shows the concentrations of elements averaged over 28 measurements in two experiments with pure hydrogen at pressures of 1 and 3.4 kbar and averaged 28 measurements in two experiments with pure helium at pressures of 1.1 and 3.05 kbar. Solid structures with "foreign" chemical elements are not formed at irradiation of metal samples without gases, including irradiation of palladium with deuterium at a pressure of 60 bar.

The most impressive result in the experiment with helium at a pressure of 1.1 kbar was the detection of thin, cylindrical, black foils of considerable dimensions in the inner part of the reaction chamber. The foils consisted predominantly of carbon. A new mechanism was proposed to explain the appearance of synthesized elements in paper [10]: multinuclear reactions. These reactions are caused to the creation of nuclear molecules, which consist of several helium nuclei $n \cdot{ }_{2}^{4} \mathrm{He}$ (Chapter 6). Nuclear molecules are created by fusion of several orthohelium atoms formed as a result of ionization of helium atoms by gamma radiation
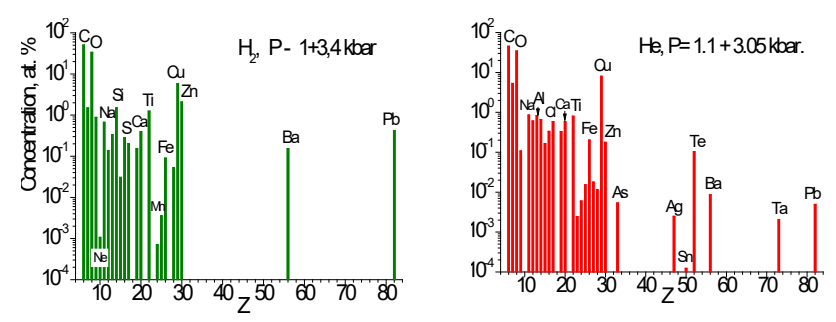

Fig. 10. Elements concentration in experiments with $\mathrm{H}_{2}$ and $\mathrm{He}$. 
followed by recombination of helium ions with electrons.

For other gases: hydrogen, deuterium, xenon, as well as for helium, it should be assumed that their ionization in the condensed medium results in formation of "capsules" with a strong magnetic field inside, which leads to low energy multinuclear reactions.

- The same paper [10] proposed to try to detect, by spectroscopic methods, the odd transmolecules using an electric discharge in a helium-hydrogen mixture at a pressure of several bar. For example, if a proton is added to $"{ }^{8} B e "$ molecule, a "boron ${ }^{9} B$ " transmolecule is formed, and the proton association with "12 $C^{\prime \prime}$ transmolecule forms the "nitrogen ${ }^{13} \mathrm{~N} "$ transmolecule. Such transmolecules are hydrogen-like atoms with heavy multinuclear formations in the center. An external electron in odd transmolecules moves along the orbital in a strong magnetic field, which is created by coupled electrons. For this reason, the external electron orbitals of transmolecules ${ }^{9} B$ " and "13 $N "$ are displaced against orbitals of hydrogen atom in accordance with corrections for reduced masses in the Rydberg constant and are split into two sublevels. Taking into account the correction in magnetic field value (an increase by 27 times, Chapter 3) related to energy of the oscillatory motion of electrons, the level splitting into the orbitals $n=2$ (the Balmer series) amounts to $(0.8-1.1) \cdot 10^{-2} \mathrm{eV}$. In accordance with these values, and taking into account that external electron in odd transmolecules moves in a strong, but inhomogeneous magnetic field, the level splitting can be observed in the form of a significant broadening of transition lines. [17]. The lines of electron transitions in the hydrogen atom located in the strong and ultrastrong magnetic fields of orthohelium and "8e" transmolecule will also broaden.

In the work of R.Mills and P.Ray on extreme ultraviolet spectroscopy of helium-hydrogen plasma [17], new emission lines with an energy higher than $27.2 \mathrm{eV}$ were observed in addition to a significant broadening of the hydrogen lines

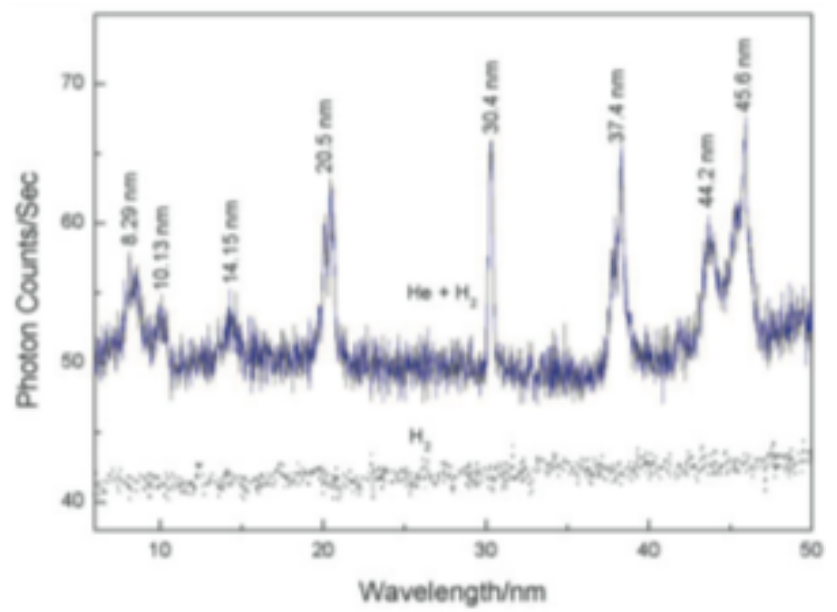

Fig. 11. Short-wave spectrum in $\mathrm{He}+\mathrm{H}_{2}$ and $\mathrm{H}_{2}$ plasmas [17].

Table 2.

Energy of lines in spectrum $\mathrm{He}+\mathrm{H}_{2}$.

\begin{tabular}{|l|l|l|l|l|l|l|l|l|}
\hline$\lambda, \mathrm{nm}$ & 8.29 & 10.13 & 14.15 & 20.5 & 30.4 & 37.4 & 44.2 & 45.6 \\
\hline $\mathrm{E}, \mathrm{eV}$ & 149.6 & 122.4 & 87.6 & 60.5 & 40.8 & 33.16 & 28.05 & 27.2 \\
\hline
\end{tabular}

in the Balmer series (Fig. 11, Table 2). Energy $2 \cdot 13.6 \mathrm{eV}$ is twice the ionization energy of the atom hydrogen. Those lines were registered at microwave discharge in a helium mixture with $2 \%$ of hydrogen at room temperature and at pressures from 20 to 1 torr. They appear in a mixture of helium and hydrogen only, but are absent in pure helium or hydrogen and in mixtures of hydrogen with other noble gases.

Since orthohelium atoms are produced in the helium plasma, there can be formed $\mathrm{He}$ transatoms

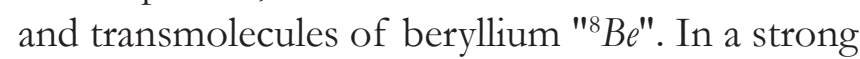
magnetic field of orthohelium $B_{S}^{R_{2}} \sim 70 T$ and in ultrastrong magnetic fields of transhelium $B_{S}^{R}(\mathrm{He})$ $\sim 7 \cdot 10^{3} T$ and of transmolecule "8 $B e^{\prime \prime} B_{S}^{R}(B e) \sim 10^{5}$ $T$, two hydrogen atoms $2 H$ or the hydrogen molecule $\mathrm{H}_{2}$ can form a "helium-pp" transmolecule (Fig. 6b). It has orthoboson-paired electrons with a lower energy level of $326.4 \mathrm{eV}$ or $163.2 \mathrm{eV}$ for each electron.

The transition of the electron orthoboson from the state of two hydrogen atoms $2 H(2 \cdot 13.6$ $\mathrm{eV})$ or the hydrogen molecule $H_{2}(2 \cdot 13.6+4.5=$ $31.7 \mathrm{eV}$ ) to the lower level of $326.4 \mathrm{eV}$ can occur either directly or as cascade. The orthoboson will emit two photons at each transition. Therefore, photons $(326.4-27.2) / 2=149.6 \mathrm{eV}$ will be emitted at direct transition from the state of the $2 \mathrm{H}$ atoms to the lower level, and photons 
$(326.4-31.7) / 2=147.4 \mathrm{eV}$ or $8.41 \mathrm{~nm}$ will be emitted at direct transition from the state of the $\mathrm{H}_{2}$ molecule (Fig. 12a). When passing through the level of hydrogen transmolecule " $H_{2}$ " from the $2 \mathrm{H}$ and $\mathrm{H}_{2}$ states, photons can be emitted in series, in pairs with energies of $87.6 \mathrm{eV}$ and $60.5 \mathrm{eV}$, giving in the sum $148.1 \mathrm{eV}$. In Chapter 5 , the level of hydrogen transmolecule is roughly estimated at $116 \mathrm{eV}$. Experimentally, the energy of this level is either $102.7 \mathrm{eV}\left(R_{\mathrm{TH}}=1.4 \cdot 10^{-11}\right.$ $m)$, or $75.6 \mathrm{eV}\left(\mathrm{R}_{\mathrm{TH}}=1.9 \cdot 10^{-11} \mathrm{~m}\right)$. This difference is not surprising, because the configuration of electrons paired to orthobosons represents a toroid (Fig. 6a), and not overlapping electron spheres (Fig. 5a)

The oscillating electron of hydrogen atom emits a photon with an energy of 2·13.6 $=27.2$ $\mathrm{eV}$ from the ground state, and if an electron is recombined with an $\mathrm{H}^{+}$ion $-3 \cdot 13.6=40.8 \mathrm{eV}$ (Fig. 12b). Hydrogen transatoms " $H$ " are formed in these cases. Photons $(326.4-81.6) / 2=122.4 \mathrm{eV}$ will be emitted in the transition from the state of two transatoms of hydrogen $2 " H " H^{\prime \prime}(2 \cdot 40.8=81.6$ $\mathrm{eV})$ to the lower level of helium transmolecule. Photons $(81.6-16.2) / 2=32.7 \mathrm{eV}(37.9 \mathrm{~nm})$ will be emitted, when electron with molecular hydrogen ion $H_{2}^{+}(13.6+2.6=16.2 \mathrm{eV})$ is recombined with the formation of two hydrogen transatoms $2 " H^{\prime \prime}$. Taking into account that in a ultrastrong magnetic field, for example: in that of transhelium $B_{S}^{R}(H e)$ $\sim 7 \cdot 10^{3} T$, the ground state of the hydrogen atom splits into two levels with energy between them

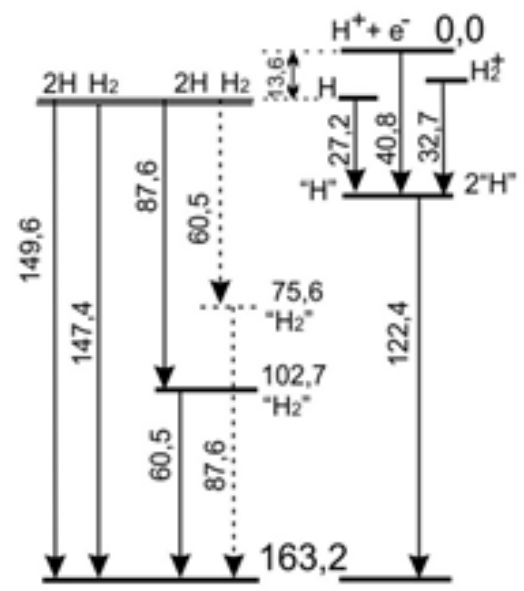

Fig. 12. Scheme of electron transitions in the bydrogenbelium transmolecule in $\mathrm{He}+\mathrm{H}_{2}$ plasma.
$\Delta E=2 \mu_{\mathrm{e}} \cdot B_{S}^{R} \sim 0.8 \mathrm{eV}$, then in the spectrum there will be observed two broad peaks 27.2 and $28 \mathrm{eV}$ (Fig. 11, Table 2).

Since the electron orthoboson is located in a ultrastrong magnetic field and its spin is $S=1$, its transition lines will be broadened and they will have a triplet structure $m_{\mathrm{S}}=+1,0,-1$ and hyperfine structure $I \neq 0$.

The formation of a "helium-pp" transmolecule will obviously lead to a nuclear reaction with a yield of a deuteron $d$ (18). Consequently, deuterium atoms appear in the discharge volume. If hydrogen is changed, in a mixture with helium, for deuterium or tritium, then neutrons will be produced in a microwave discharge by reactions $(20,21)$ and ${ }^{6} \mathrm{Li}(671 \mathrm{~nm}$, red light) according to reaction (22).

Only helium, namely, orthohelium with its own strong magnetic field is a catalytic element that creates, in a mixture with hydrogen, conditions for formation of hydrogen-"helium" transmolecule and for realization of nuclear fusion reaction of two protons and an electron. Other inert gases do not create strong magnetic fields.

- It can not be excluded that in experiments [18], conducted in the fifties of the last century under the leadership of I. Kurchatov and investigating the possibility of triggering thermonuclear reactions in the gas environment of hydrogen, deuterium, helium, and their mixtures, the appearance of neutrons and powerful $X$-rays in the energy range 300 $400 \mathrm{keV}$ was connected with non-Coulomb multinuclear processes.

On February 15, 2018, T.J. McCuire on behalf of the company Lockheed Martin received a patent: "Encapsulating magnetic fields for plasma confinement" [19]. The company filed that patent on April 2, 2013. In October, 2014, the corporation said that preliminary research results indicate the possibility of creating reactors operating on fusion of light nuclei with a power of about 100 megawatts and dimensions comparable to a truck, which is about ten times smaller than existing thermonuclear fusion installations. Scientists engaged in research in the 
field of controlled thermonuclear fusion called the Lockheed Martin message at that time erroneous, since it is impossible to create and confine a hightemperature plasma in such a volume. Therefore, it is quite probable that not thermonuclear reactions take place in the LM reactor, but fusion reactions of light nuclei based on non-Coulomb nuclear reactions.

\section{STELLAR ENERGY AND NUCLEOSYNTHESIS}

Taking into account all LENR experiments, it should be assumed that as a result of ionization of a weakly excited condensed matter, local, stable, electron-ion formations - "capsules" with a strong magnetic field inside them appear in that matter. Low energy nuclear reactions take place in those "capsules". It is necessary to understand the physical nature and mechanism of the appearance of "capsules" in a condensed matter. LENR reactions, namely: transmutation reactions [2], mainly occur in melts, in solutions and in dense gases, i.e. on free atoms. The conditions under which "capsules" are formed are much softer than the conditions for thermonuclear processes. Therefore, the stars and the Sun are probably not thermonuclear reactors. And obtaining energy by the method of thermonuclear fusion is not a nature-like, effective technology.

In the experiments of Solin M.I. [11, 20], Kladov A.F. [21], Didyk A.Yu. [1516] and many other LENR experiments,

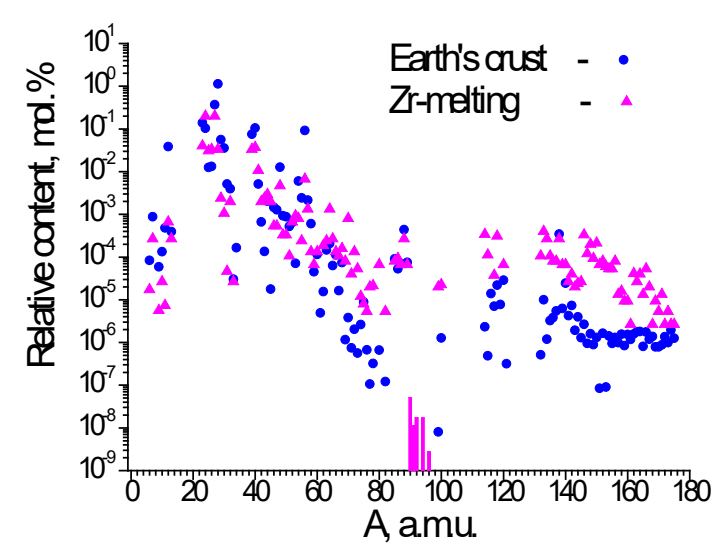

Fig. 13. Comparison of mass distribution in case of ${ }_{40} \mathrm{Zr}$ melting with element abundance in Earth's crust [20, 22].

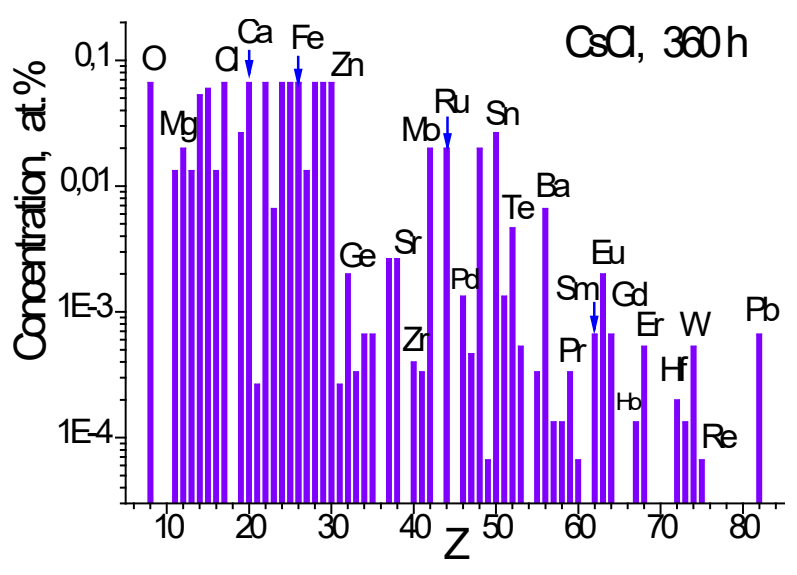

Fig. 14. Ultrasound cavitation of solution ${ }_{55} C s_{17} \mathrm{Cl}[21,24]$.

which lasted for a considerable time: from several hours to several days, the reaction products produced "practically all" elements of Periodic Table. Fig. 13 presents a comparison of mass distributions in case of zirconium transmutation ${ }_{40} \mathrm{Zr}$ [20] with elements distribution in the Earth's crust. For orientation, the zirconium isotopes are underlined by lines, in accordance with their relative content. Fig. 14 shows the yield of chemical elements in experiment [21] on ultrasonic cavitation of a $\mathrm{CsCl}$ salt solution for 360 hours. Fig. 15 gives the concentrations of chemical elements averaged over three experiments on irradiation by bremsstrahlung gamma quanta of condensed xenon ${ }_{54} \mathrm{Xe}$ at pressures of 250, 270 and 550 bar for 43 to 72 hours. Obviously, in order to receive chemical elements, including superheavy elements, it is not necessary to blow up supernova stars

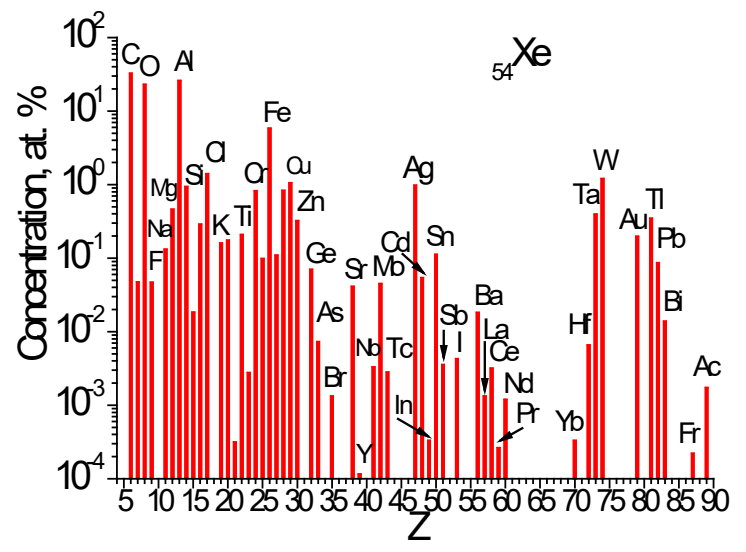

Fig. 15. Concentration of chemical elements in experiments with ${ }_{54} X e[15,16]$. 
[22-24]. Apparently, nucleosynthesis can also occur due to low energy transmutation reactions.

\section{CONCLUSION}

It was pointed out in papers $[24,25]$ that there are three theoretical prohibitions of the phenomenon of low energy transmutation of chemical elements: - impossibility to pass the Coulomb barrier;

- extremely small probabilities of weak processes;

- small probability of polyatomic collisions.

The exchange interaction between electrons in an atom in a strong magnetic field leads to their pairing with the formation of orthobosons with spin equal to unity $S=1$. Electron orthobosons form a Bose condensate, thereby transforming the atom into a transatom. Since the magnetic moments of electrons in Bose condensate are directed in one direction, they create a giant inhomogeneous and anisotropic magnetic field inside and around the transatom.

The internal magnetic field transforms atomic nuclei into transnuclei.

The external magnetic field and the electron Bose condensate of transatoms allow them to be attracted to each other, thus creating multinuclear molecules - transmolecules. Thus, the third prohibition of the small probability of "polyatomic collisions" is removed.

The exchange Coulomb energy of paired electrons has the character of attraction and it completely compensates, in the first order of perturbation theory, the energy of their Coulomb repulsion. Oscillations of paired electrons in the transatom increase by three times their binding energy and decrease by three times the radius of their orbitals. Therefore, electrons in the Bose condensate are located on the orbital closest to atomic nucleus. The wave functions of electrons in transatoms and transmolecules significantly overlap with wave functions of transnuclei.

This property of transatoms and transmolecules in low energy transmutation reactions allows us to overcome the second prohibition on small probabilities of weak processes responsible for the transformation of protons into neutrons and vice versa.

The ultrastrong internal magnetic field of transmolecules, consisting of identical transnuclei allows the latter, due to exchange interaction, to form an orthoboson. Since the exchange energy of identical transnuclei compensates for their Coulomb repulsion, they can enter into nuclear interactions. Transnuclei in the transmolecule move in inhomogeneous and anisotropic space created by an ultrastrong magnetic field. Thus, motion integrals are not conserved in the interaction of the transnuclei: momentum conservation law, angular momentum (spin) conservation law, and energy conservation law are violated. As a consequence, the non-radioactive low energy transmutation reactions automatically take place in a nuclear transmolecule.

Thus, the main prohibition on the implementation of transmutation reactions - the impossibility of passing the Coulomb barrier - is removed.

Numerous and diverse, simple and complex experiments on low energy transmutation of chemical elements demonstrate the obviousness of those processes. A further study of low energy nuclear reactions and their application create a basis for a new scientific and technological revolution.

\section{REFERENCES}

1. Mishinsky GV. Atom in a strong magnetic field. Transformation of atoms to transatoms. Radioelectronics. Nanosystems. Information Technologies (RENSIT), 2017, 9(2):147-160; DOI: $10.17725 /$ rensit.2017.9.147.

2. Mishinsky GV. Magnitnye polya transatomov. Spinovy-nuklidny-elektronny kondensat [Magnetic fields of transatoms. Spinnuclide-electronic condensate]. Zhurnal Formiruyushchkhsya napravleniy nauki (ZhFNN), 2017, 15-16(5):6-25 (in Russ.); http://www.unconv-science.org/.

3. Mishinsky GV. Transatoms - Transnuclei, and their properties. The materials of the 18th Russian 
conference on cold transmutation of nuclei of chemical elements, 2012, Moscow, pp. 94-106.

4. Matveev AN. Atomic physics. Moscow, Vysshaya shkola Publ., 1989, 439 p.

5. Heisenberg W. Über die Spektra von Atomsystemen mit zwei Elektronen. Z. Phys., 1926, 39(7):499-518.

6. Fock VA. Bemerkung zur Quantelung des harmonischen Oszillators im Magnetfeld. Z.Phys., 1928, 47:446-448.

7. Heiss WD, Nazmitdinov RG. Orbital magnetism in small quantum dots with closed shells. Pis'ma v ZhETF, 1998, 68(12):870-875 (in Russ.).

8. Landau LD, Lifshitz EM. Qauntum mechanics. Nonrelativistic theory. Moscow, Nauka Publ., 1974, 752 p.

9. Landau LD, Lifshitz EM. Field theory. Moscow, Nauka Publ., 1973, 138 p.

10. Mishinsky GV. Mnogoyadernye reaktzii V kondensirovannomgelii [Multinuclear reactions in condensed helium]. RENSIT, 2017, 9(1):94105; DOI: 10.17725/rensit.2017.9.094.

11. Balakirev VF, Krymsky VV, Bolotov BV. et.al. Vzaimoprevrashchenie khimicheskikh elementov. Ekaterinburg, Urals Branch of RAS Publ., 2003, 64 p.

12. Didyk AYu, Wisniewski R. Nuclear reactions, induced by gamma quanta, in palladium saturated with deuterium surrounded by dense deuterium gas. Euro. Phys. Lett., 2012, 99(22001):1-6.

13. Didyk AYu, Wisniewski R, WilczynskaKitowska T. The carbon-based structures synthesized through nuclear reactions in helium at 1.1 kbar pressure under irradiation with braking $\gamma$-rays of $10 \mathrm{MeV}$ threshold energy. Euro. Phys. Lett., 2015, 109(22001):1-6.

14. Didyk AYu. Nuclear reactions of fusion and fission chemical elements and the formation of new structures in dense gases $\mathrm{H}_{2}, \mathrm{D}_{2}$ and $\mathrm{He}$ at irradiation $10 \mathrm{MeV}$ of $\gamma$-quanta. Physics and chemistry of materials processing, 2015, 2:5-19.
15. Wisniewski R, Mishinsky GV, Gulbekian GG, Wilczyńska-Kitowska T, Semin VA. Synthesis of chemical elements and solid-state structures under irradiation by $\gamma$-quanta in condensed gases. ZhFNN, 2017, 17-18(5):615 (in Russ.).

16. Didyk AYu, Wisniewski R, Mishinsky GV, Semin VA, Wilczyńska-Kitowska T. Synthesis of solid-state structures and chemical elements under irradiation by bremsstrahlung $\gamma$-rays with a maximum energy of $10 \mathrm{MeV}$ in condensed deuterium at a pressure of 2.2 kbar. Preprint P15-2018-3. JINR, Dubna, 2018, $19 \mathrm{p}$.

17. Mills R, Ray P. Extreme ultraviolet spectroscopy of helium-hydrogen plasma. J. Phys. D:Appl. Phys., 2003, 36:1535-1542.

18. Kurchatov IV. About possibility of creation of thermonuclear reactions in gas discharge. UFN, 1956, 59(4):603-618.

19. Lockheed Martin Corporation. McCuire TJ. Encapsulating magnetic fields for plasma confinement. Patent US2018/0047462 A1, feb.15, 2018.

20. Solin MI. Experimental facts of spontaneous appearance of condensate of soliton charges with the formation of nuclear fusion products in liquid zirconium. Physical Idea in Russia, 2001, No. 1, pp. 43-58. (in Russ.).

21. Kladov AF. Cavitation destruction of matter (in Russ.); http://roslo.narod.ru/rao/rao1. htm.

22. Mishinsky GV, Kuznetsov VD, Penkov FM. To the question of mechanism of synthesis of chemical elements at irradiation of condensed gases by bremsstrahlung gamma quanta. ZhFNN, 2017, 17-18(5):45-57 (in Russ.).

23. Mishinsky GV, Kuznetsov VD. Element distribution in the products of low energy transmutation. Nucleosynthesis. Annales de la Fondation Louis de Broglie, 2008, 33(3-4):331-356.

24. Mishinsky GV, Kuznetsov VD, Penkov FM. Low energy transmutation of atomic nuclei of chemical elements. Element distribution 
in the products of low energy transmutation. Nucleosynthesis. ZhFNN, 2017, 17-18(5):6181 (in Russ.).

25. Kuznetsov VD, Mishinsky GV, Penkov FM, Arbuzov VI, Zhemenik VI. Low energy transmutation of atomic nuclei of chemical elements. Annales de la Fondation Louis de Broglie, 2003, 28(2):173-214. 